\title{
1 Metastable GPCR dimers trigger the basal signal by recruiting
}

\section{G-proteins}

5 Rinshi S. Kasai ${ }^{1}$, Takahiro K. Fujiwara², and Akihiro Kusumi3*

$9 \quad{ }^{1}$ Institute for Frontier Life and Medical Sciences and 2 Institute for Integrated Cell-Material

10 Sciences, Institute for Advanced Study, Kyoto University, Kyoto 606-8507, Japan.

11 3Membrane Cooperativity Unit, Okinawa Institute of Science and Technology Graduate

12 University (OIST), Onna-son, Okinawa, 904-0495 Japan.

$17 *$ To whom all correspondence should be addressed:

18 Akihiro Kusumi

19 e-mail: akihiro.kusumi@oist.jp

20 Ph: 011-81-98-966-1373, Fx: 011-81-98-966-1062

21

22 
G-protein-coupled receptors (GPCRs) constitute the largest family of integral membrane proteins in the human genome and are responsible for various important signaling pathways for vision, olfaction, gustation, emotion, cell migration, etc. A distinct feature of the GPCR-family proteins is that many GPCRs, including the prototypical GPCR, $\beta 2$-adrenergic receptor ( $\beta 2 A R)$, elicit low levels of basal constitutive signals without agonist stimulation, which function in normal development and various diseases $^{1-3}$. However, how the basal signals are induced is hardly known. Another general distinctive feature of GPCRs is to form metastable homo-dimers, with lifetimes on the order of $0.1 \mathrm{~s}$, even in the resting state. Here, our single-molecule-based quantification $^{4}$ determined the dissociation constant of $\beta 2 A R$ homo-dimers in the PM $\left(1.6 \pm 0.29\right.$ copies $\left./ \mu \mathrm{m}^{2}\right)$ and their lifetimes $(83.2 \pm 6.4 \mathrm{~ms})$, and furthermore found that, in the resting state, trimeric G-proteins were recruited to both $\beta 2 A R$ monomers and homo-dimers. Importantly, inverse agonists, which suppress the GPCR's basal constitutive activity, specifically blocked the G-protein recruitment to GPCR homo-dimers, without affecting that to monomers. These results indicate that the G-proteins recruited to transient GPCR homo-dimers are responsible for inducing their basic constitutive signals. These results suggest novel drug development strategies to

B2AR forms metastable homo-dimers

Single-molecule imaging-tracking revealed that $\beta 2 \mathrm{AR}$ (tagged with ACP at the N-terminus and labelled with ATTO594; Extended Data Fig. 1) undergoes thermal diffusion in the plasma membrane (PM), and often forms transient homo-dimers, undergoing rapid interconversions between monomers and dimers continually, as found for other GPCRs (Fig. 1a, Extended Data Fig. 2a $)^{4-7}$. Every time we found a $\beta 2 A R$ dimer, we measured the dimer duration, and after observing many dimers, we obtained the distribution of dimer durations (Fig. 1b, Extended Data Fig. 2d). The histogram could be fitted by a single exponential function, providing the exponential dimer lifetime of $99 \mathrm{~ms}$ (throughout this report, all statistical parameters, including SEM, and the 
51 number of experiments are summarised in Extended Data Tables 1-4; all observations were

52 performed at $37^{\circ} \mathrm{C}$ ). The lifetime value obtained in this manner, termed $\tau_{\text {observed, }}$ was corrected for

53 the photobleaching lifetime and incidental colocalisation lifetime (this method was used for

54 evaluating all of the lifetimes investigated in the present research; see Methods), giving a dimer

55 lifetime $\tau$ of $83.2 \pm 6.4 \mathrm{~ms}$ (Fig. 1C). The dimer lifetime obtained at a time resolution of $4 \mathrm{~ms}$ (250

$56 \mathrm{~Hz}$ ) was $78.8 \pm 10.7 \mathrm{~ms}$, after corrections for photobleaching and incidental colocalisation lifetime

57 (Extended Data Fig. 3, Fig. 1c), which is statistically indistinguishable from the dimer lifetime of

$58 \quad 83.2 \mathrm{~ms}$ evaluated from $30-\mathrm{Hz}$ observations. This result indicates that a frame rate of $30 \mathrm{~Hz}$ is

59 sufficiently fast for evaluating the homo-dimer lifetime of $\beta 2 A R$. Together, these results demonstrate

60 that the $\beta 2 A R$ homo-dimers are indeed quite metastable, turning into monomers in a matter of 80

$61 \mathrm{~ms}$, and then quite readily forming another dimer with a different partner molecule (Extended

62 Data Fig. 2a).

\section{D-dissociation constant for $\beta 2 A R$ dimers}

65 Using single-molecule-based "super-quantification" method previously developed by us ${ }^{4}$, we obtained the 2-dimensional dissociation constant (2D- $\left.K_{D}\right)$ of $\beta 2 A R$ homo-dimers in the PM of living cells, which was $1.6 \pm 0.29$ copies $/ \mu m^{2}$ (Fig. 2a, Extended Data Fig. 2b; see Methods). This value is comparable to the $2 \mathrm{D}-K_{\mathrm{D}}$ of another GPCR, formyl-peptide receptor (FPR), which was 3.6 copies $/ \mu \mathrm{m}^{2}$ (in fact, this was the first determination of the $2 \mathrm{D}-K_{\mathrm{D}}$ in the PM of living cells) ${ }^{4}$.

Under physiological conditions, $\beta 2 A R$ is expressed at number densities of $16 \sim 260$ copies $/ \mu \mathrm{m}^{2}$,

71 depending on the cell type ${ }^{8,9}$. From the 2D- $K_{\mathrm{D}}$ determined here, we conclude that $12 \sim 240$ copies $/ \mu \mathrm{m}^{2}$ exist as dimers and $4 \sim 20$ copies $/ \mu \mathrm{m}^{2}$ exist as monomers at any moment; i.e., $75 \sim$ $92 \%$ of $\beta 2 A R$ molecules exist as homo-dimers at any moment, although dimers and monomers are continually interconverting rapidly all the time (with a dimer lifetime of $\sim 80 \mathrm{~ms}$ ). In contrast, FPR is mainly expressed in neutrophils, and an average of about 6,000 copies of FPR copies exist in the PM of a single neutrophil ${ }^{10}$. Assuming that the neutrophil surface area is $1,300 \mu \mathrm{m}^{2}$ (approximated by a sphere with a radius of $10 \mu \mathrm{m}$, providing a number density of $\sim 4.6$ FPR copies/ $\mu \mathrm{m}^{2}$ ) and based on 
as dimers; namely, 54\% of FPR molecules are dimers, which is about two-thirds of the dimer fraction of $\beta 2 A R$. Note that, even under the conditions where the dimer population dominates (i.e., at higher expression levels), the dimer lifetimes do not change and remain on the order of $0.1 \mathrm{~s}$. Only the monomer lifetimes are shortened at higher expression levels.

\section{Direct detection of $\beta 2 A R$ dimers by the BiFC method}

Thus far, we have detected dimers by the colocalisation of two single molecules, which reveals the close encounters of molecules in the space scale of $220 \mathrm{~nm}$, a distance much greater than the molecular scale. Therefore, in this method, the incidental approaches of two molecules were evaluated and subtracted. To ascertain the molecular-level interactions (dimers), we employed YFP-based bimolecular fluorescence complementation (BiFC; meanwhile, our attempt to detect

FRET did not work, perhaps because the distance between the probes attached to two C-termini was too far for FRET) ${ }^{4,11}$. In BiFC, two potentially interacting proteins are fused to the $\mathrm{N}$ - and C-terminal half-molecules of YFP. If the proteins of interest form dimers, then YFP may be reconstituted and emit fluorescence signals (see Methods). Thus, if BiFC occurs, it would lend strong support for the formation of $\beta 2 A R$ dimers.

To quantitatively examine BiFC, we varied the expression levels of $\beta 2 A R$. With an increase in the number density of expressed molecules (determined by the signal from ATTO594 bound to ACP; the ATTO594/ACP ratio is $>0.95^{12}$ ), the number density of the BiFC spots of $\beta 2 A R$ increased, whereas the BiFC-spot number density of the monomer reference molecule, ACP-TM, increased only slightly (Fig. 2b; Extended Data Fig. 2c). This result indicates that $\beta 2 A R$ forms directly-bound dimers.

\section{Inverse agonists reduce the $\beta 2 A R$ dimer affinity}

103 Treatments with the inverse agonists ICI-118,551 (ICI) and timolol reduced the $\beta 2$ AR dimer lifetime 104 (0.74x and 0.69x, respectively; Fig. 1C, Extended Data Fig. 2d), whereas ICI increased the 2D- $K_{D}$ of $\beta 2 A R$ by a factor of 2.1 (lowered the $\beta 2 A R$ dimer affinity; Fig. 1c), indicating that inverse agonists reduce the $\beta 2 A R$ dimer affinity. Meanwhile, the addition of the agonist isoproterenol (Iso) prolonged 
107 the dimer lifetime significantly (1.55x) (Fig. 1C; Extended Data Fig. 2d). Cholesterol reportedly

108 modulates the functions of some $\mathrm{GPCRs}^{13}$, but here we found that mild cholesterol depletion by 109 methyl- $\beta$-cyclodextrin (M $\beta C D$ ) treatment failed to affect the $\beta 2 A R$ dimer lifetime (Fig. 1c).

Since the inverse agonists suppressed the basal constitutive GPCR signaling, and since we

111 found that they also reduced the $\beta 2 A R$ dimer lifetime, we next examined the dimer lifetimes of the

$112 \beta 2 A R$ mutants with high and low basal activities in the resting state (HBA and LBA mutants,

113 representing E268A and C327R mutants, respectively $\left.{ }^{14,15}\right)$. The HBA mutant exhibited a 1.78x longer

114 dimer lifetime, and interestingly, after the inverse-agonist addition, it was reduced to the dimer

115 lifetime of the wild-type receptor (Fig. 1c; Extended Data Fig. 2d). Meanwhile, the LBA mutant

116 exhibited a $0.74 x$ shorter dimer lifetime than that of the wild-type receptor, and the agonist addition 117 did not affect it (Fig. 1c; Extended Data Fig. 2d).

118 Strikingly, the dimer lifetimes of WT- $\beta 2$ AR after the addition of inverse agonists were quite 119 comparable to those of the LBA mutant, and the dimer lifetime of WT- $\beta 2$ AR after the addition of the 120 agonist isoproterenol was comparable to that of the HBA mutant. These results suggest that the $121 \quad \beta 2 A R$ conformations would be quite different after the binding of the inverse agonists and the 122 agonist isoproterenol, as compared with those before their additions ${ }^{16}$, possibly resembling the 123 conformations of the LBA and HBA mutants, respectively, and detectable as changes in the 124 homo-dimer lifetimes.

The basal constitutive activity of $\beta 2 A R$

127 We confirmed the basal constitutive activity of $\beta 2 A R$, as well as the effects of the $\beta 2 A R$ agonist 128 isoproterenol and the inverse agonists ICI-118,551 and timolol, by measuring the changes in the 129 cytoplasmic cAMP levels in the L-cell line stably expressing $\beta 2 A R$ at higher levels (Extended Data 130 Fig. 4, Extended Data Table 4). The addition of the agonist isoproterenol to the cells increased 131 the cAMP level by a factor of $\sim 4$, relative to the control. Meanwhile, the addition of the inverse 132 agonists ICI-118,551 and timolol reduced the cAMP levels by factors of 1.9 and 1.3 (53 and 76\% of 133 the control), respectively, showing the presence of the basal constitutive activity of $\beta 2 A R$ and the effectiveness of the inverse agonists, ICI-118,551 and timolol, for strongly reducing this basal 
activity.

\section{Trimeric G proteins are recruited to both $\beta 2 A R$ monomers and dimers}

138 The presence of the basal constitutive activity of $\beta 2 A R$ suggests that a stimulatory trimeric G-protein 139 might be recruited to $\beta 2 A R$ in the resting state ${ }^{17}$. Therefore, we examined whether $\mathrm{G} \alpha$ s and $\mathrm{G} \beta$

140 (conjugated with mGFP) are recruited to $\beta 2 A R$ monomers or dimers, by examining their

141 colocalisations. Using L cells expressing both $\beta 2 A R$ (ATTO594-ACP- $\beta 2 A R$ ) and Gas or G $\beta$, we found 142 that, even in resting cells, Gas and G $\beta$ were recruited to both $\beta 2 A R$ monomers and dimers (Fig. 3a;

\section{Supplementary Videos 1 and 2).}

144 The recruitment frequencies of $\mathrm{G} \alpha \mathrm{s}$ and $\mathrm{G} \beta$ to $\beta 2 \mathrm{AR}$ monomers and dimers were measured 145 and then normalised by the number densities of $\mathrm{G} \alpha \mathrm{s}$ (or $\mathrm{G} \beta \gamma$ ) and $\beta 2 \mathrm{AR}$ protomers in the PM 146 (normalised frequencies, with a dimension of per sec per $\mu \mathrm{m}^{4}$; Fig. 3b, c; Extended Data Table $1473)^{18}$. In resting cells, both $\mathrm{G} \alpha \mathrm{S}$ and $\mathrm{G} \beta$ molecules were recruited to $\beta 2 A R$ monomers or dimers 148 equally well, suggesting that (non-stimulated) $\beta 2 A R$ monomers and/or dimers might induce 149 intracellular Gs-dependent signals even in the resting state, which would lead to the basal 150 constitutive activity of $\beta 2 A R$.

The exponential dwell lifetimes of $\mathrm{G}$ proteins on $\beta 2 A R$ were on the order of $50 \sim 60 \mathrm{~ms}$ for all of the combinations of $\mathrm{G} \alpha \mathrm{s}$ and $\mathrm{G} \beta$ molecules vs $\beta 2 \mathrm{AR}$ monomers and dimers (Extended Data Fig. 5). However, these durations are only the maximal estimates of the dwell lifetimes of G $\alpha$ S and G $\beta$ molecules on $\beta 2 A R$ monomers and dimers, due to the present instrumental limitations of detecting short binding durations.

Notably, the treatment of the cells with the agonist, isoproterenol, did not change the recruitment frequencies in any combinations of $G \alpha s$ and $G \beta$ vs $\beta 2 A R$ monomers and dimers,

158 although it significantly enhanced the cytoplasmic cAMP level (Extended Data Fig. 4, Extended

159 Data Table 4). This result is quite surprising, but it suggests that the isoproterenol-induced $\beta 2 A R$ 160 conformation enhanced its GEF activity, rather than increasing the on-rate of G protein binding to $161 \beta 2 A R$. 


\section{Inverse agonists specifically block G-protein recruitment to $\beta 2 A R$ dimers}

164 Next, we examined the effects of the inverse agonists. The recruitment of G $\alpha$ s and G $\beta$ to $\beta 2 A R$ dimers was almost completely blocked, whereas that to $\beta 2 A R$ monomers was unaffected (Fig. $\mathbf{3 b}$, c). This result unequivocally shows that $\beta 2 A R$ dimers are responsible for the basal constitutive activity of $\beta 2 A R$. Furthermore, it demonstrates that the inverse agonists work by blocking the recruitment of stimulatory G-proteins to $\beta 2 A R$ dimers, perhaps by inducing $\beta 2 A R$ conformational changes in the binding sites for G-proteins, rather than blocking the $\beta 2 A R$ dimerisation (although, as already described, the dimer affinity of the inverse agonist-bound $\beta 2 A R$ is lower, resembling the LBA mutant, and thus inverse agonists might change the GPCR conformations in two ways: lowering the GPCR dimer affinity and modulating the G-protein binding sites).

To further confirm that $\beta 2 A R$ dimers trigger downstream signaling without an agonist, and to detect the basal dimer signal at very low $\beta 2 A R$ expression levels used for single-molecule experiments (generally $<1 \mathrm{copy} / \mu \mathrm{m}^{2}$, which is approximately comparable to $<3,000$ copies in the $\mathrm{PM} /$ cell), we examined whether artificially-induced stable $\beta 2 A R$ dimers could elicit the downstream signal. To detect the downstream signal at very low expression levels of $\beta 2 A R$, which are necessary

178 for detecting dimers by single-molecule imaging, and also to detect the signal in a different manner, rather than observing the CAMP level again (Extended Data Fig. 4), we examined the intracellular $\mathrm{Ca}^{2+}$ mobilisation, which could occur further downstream from the cAMP amplification ${ }^{19}$. The induction of the artificially-induced stable $\beta 2 A R$ (conjugated to FKBP) dimers by the FKBP-A20187 dimerisation system indeed elicited $\mathrm{Ca}^{2+}$ mobilisation (Extended Data Fig. 6). This result further confirmed that $\beta 2 A R$ dimers could trigger the downstream signals without agonist stimulation.

\section{Discussion}

All of the class-A GPCRs examined thus far, including $\beta 2 A R$ examined here and by others ${ }^{5}, F P R, D 2$ dopamine receptor, $\mathrm{M} 1$ muscarinic receptor, and $\alpha 1 \mathrm{AR}$, form metastable dimers with lifetimes on the order of $0.1 \mathrm{~s}$ in the non-stimulated state ${ }^{4-7}$. Therefore, we propose that the dynamic equilibrium between monomers and homo-dimers is a common characteristic property of the class-A GPCRs. 
191 through evolution, GPCRs changed almost all of their amino acids, but conserved the physical ability

192 to form transient homo-dimers. This infers that these dynamic dimers are critical for some of the 193 GPCR functions.

194 GPCRs are distinct among other receptors, in that, under non-stimulated conditions, GPCRs

195 maintain the low basal constitutive activity, which can be inhibited by inverse agonists. The basal 196 activities of GPCRs are implied in normal development and various diseases ${ }^{1-3}$. Therefore, we 197 examined the possibility that the function of the GPCR dimers is to induce the basal constitutive 198 activity in resting cells (without agonists), and obtained three unexpected, surprising findings. First, 199 both $\beta 2 A R$ monomers and dimers continually recruit $G$ proteins, one molecule after another, even in 200 the resting state. Second, the G-protein recruitment frequencies in the resting state are about the 201 same in the presence of the agonist. Third, more importantly, the inverse agonists specifically and 202 almost completely blocked the recruitment of $\mathrm{G} \alpha \mathrm{S}$ and $\mathrm{G} \beta$ to $\beta 2 A R$ dimers, without affecting their 203 recruitment frequencies to $\beta 2 A R$ monomers (Fig. 3b, c). The third result unequivocally shows that 204 the G proteins bound to $\beta 2 A R$ dimers, and not monomers, in the resting state are responsible for 205 triggering the $\beta 2 A R^{\prime}$ s basal constitutive activity, which is sensitive to inverse agonism. It further 206 suggests that the binding of the inverse agonists to $\beta 2 A R$ induces conformational changes that 207 decrease the $\beta 2 A R$ dimer affinities for $G$ proteins (Fig. 2a) and at the same time, hide the binding 208 site(s) for $\mathrm{G}$ proteins in the dimer conformations.

209 Although the function of the GPCR dimers is to maintain the basal constitutive activity, it is 210 critically important to realise that the GPCR dimers are metastable with lifetimes on the order of 0.1 211 s. Therefore, the GPCR dimers are dispersing continually, forming new dimers with other partner 212 molecules all the time. In cells, this allows the basal constitutive activities of GPCRs to be maintained 213 at certain levels, and yet the levels of the basal GPCR signals (the average number density of GPCR 214 dimers at any time) are regulated globally, by modulating the GPCR number density by varying the 215 expression levels of the GPCR, and locally, by temporarily and spatially varying the actin-based 216 membrane skeleton and the membrane curvature ${ }^{5,20-22}$. In addition, the metastable GPCR dimers 217 might play important roles in the activation of $\mathrm{G}$ proteins after the agonist addition. 
219 performed by the basal activities induced by their homo-dimers, as the GPCR family includes many

220 orphan receptors ${ }^{3,23}$. Based on the results described in this report, we strongly suggest the pursuit of

221 drug development strategies that emphasise the generation of more inverse agonists for various

222 GPCRs, as well as drugs that enhance or suppress GPCR homo-dimer formation.

223

224 Online content

225 Any methods, additional references, Nature Research reporting summaries, source data, extended 226 data, supplementary information, acknowledgements, peer review information; details of author

227 contributions and competing interests; and statements of data and code availability are available at 228 http://doi.org/????????. 


\section{Acknowledgements}

231 We thank Prof. Hajime Fujisawa of Nagoya University for providing mouse L cells, Prof.

232 Tatsuya Haga of Gakushuin University for providing the cDNAs encoding $\beta 2 A R$ and $G \alpha$, Prof.

233 Tobias Meyer of Stanford University for providing the cDNA encoding YFP-FKBP through 234 Addgene (plasmid 201751), Prof. Michiyuki Matsuda of Kyoto University for the excellent 235 suggestion about the method for establishing cell lines stably expressing target proteins at 236 high levels, Assoc. Prof. Akira Hattori for the use of the PerkinElmer time-resolved 237 fluorescence resonance energy transfer reader, EnVision Xcite, for the cAMP measurement, 238 Drs. Taka-Aki Tsunoyama of OIST, Ko Hirosawa of Gifu University, Prof. Kenichi Suzuki of Gifu 239 University, and all of the members of the Kusumi laboratory for valuable discussions, 240 suggestions, and support, and Mr. Koji Kanemasa of incomings for assistance with figure 241 preparation. This work was supported by Grants-in-Aid for Scientific Research from the 242 Japan Society for the Promotion of Science (Kiban C to R.S.K. [17K07333], Wakate B to R.S.K. [26870292], Kiban S to R.S.K. [Co-PI, 18H05269], and Kiban B to R.S.K. [Co-PI, 244 17H03666], Kiban B to T.K.F. [16H04775], and Kiban S to A.K. [16H06386]), Grants-in-Aid 245 for Innovative Areas (Molecular Engine) from the Ministry of Education, Culture, Sports, Science, and Technology (MEXT) of Japan to R.S.K. [18H05424], and the Joint Research

247 Program of Biosignal Research Center of Kobe University to R.S.K. [301002]. WPI-iCeMS of Kyoto University is supported by the World Premier Research Center Initiative (WPI) of the 249 MEXT.

\section{Author contributions}

R.S.K. performed the single fluorescent-molecule tracking experiments and biochemical experiments. R.S.K., T.K.F., and A.K. developed and built the single-molecule imaging station. T.K.F. developed the analysis software. R.S.K. and A.K. conceived and formulated the project, evaluated and discussed the data, and wrote the manuscript, and all authors participated in revising the manuscript. 


\section{Methods}

260 Cell culture, cDNA construction and expression in L cells, and treatment of cells 261 with agonists and inverse agonists.

262 L cells (a kind gift from $H$. Fujisawa of Nagoya University), which do not express $\beta 2 A R^{24}$, were confirmed to be free of mycoplasma contamination using MycoAlert (Lonza), and were routinely cultured in Dulbecco's Minimum Essential Medium (DMEM; Sigma-Aldrich) supplemented with 10\% (v/v) FBS (Sigma-Aldrich), 100 units/ml penicillin (Wako), and 0.1 $\mathrm{mg} / \mathrm{ml}$ streptomycin (Wako). Human $\beta 2 A R$ was fused at its $\mathrm{N}$-terminus to the ACP- or Halo7-tag protein (NEB and Promega, respectively) at the cDNA level, and cloned into the expression plasmid pEGFP-N1 (Clontech) after the removal of the cDNA region encoding EGFP. The ACP- and Halo7-tag proteins do not induce dimerisation ${ }^{11,25}$. For the genetic conjugation of mGFP to Gas, the mGFP cDNA was inserted between the region encoding the 271 71st and 82nd amino acids of mouse Gas (Origene) in the pcDNA3.1 (+) vector 272 (Invitrogen) ${ }^{26}$, and for that to $\mathrm{G} \alpha, \mathrm{mGFP}$ was fused to the $\mathrm{N}$ terminus of bovine $\mathrm{G} \alpha 1$ (a kind 273 gift from T. Haga of Gakushuin University) ${ }^{27}$ in the pEGFP-C1 vector (Clontech), after the 274 removal of the cDNA region encoding EGFP. The details of the constructed cDNAs are shown 275 in Extended Data Fig. 1. The L cells were transfected with these cDNAs using 276 LipofectAMINE LTX (Life Technologies), according to the manufacturer's recommendations. 277 Transfected cells were seeded in glass-base dishes (35 $\mathrm{mm} \phi$ with a glass window of $12 \mathrm{~mm} \phi$, 278 0.12 0.17-mm-thick glass; Iwaki), and cultured in Ham's F12 medium (Sigma-Aldrich), 279 supplemented with $10 \%$ (v/v) FBS (Sigma-Aldrich), 100 units/ml penicillin (Wako), and 0.1 $280 \mathrm{mg} / \mathrm{ml}$ streptomycin (Wako), for 24 48 $\mathrm{h}$ before microscope observations. The F12 medium 281 was employed because it has a lower fluorescence background as compared with DMEM, 282 which was used for routine culturing.

284 (Tocris) and timolol (Wako), were dissolved in Hank's balanced salt solution (Nissui) 285 buffered with 2 mM piperazine-N,N'-bis(ethanesulfonic acid) (PIPES, Dojindo) at pH 7.4 
286 (P-HBSS), at concentrations of 20, 200, and $200 \mu \mathrm{M}$, respectively. For the additions to the

287 cells, $1 \mathrm{ml}$ solutions of these reagents were added to the cells in $1 \mathrm{ml}$ of the Ham's F12

288 medium described above, providing the final concentrations of $10 \mu \mathrm{M}$ isoproterenol, $100 \mu \mathrm{M}$ 289 ICI-118,551, and $100 \mu \mathrm{M}$ timolol.

291 Labelling of $\beta 2 A R$ with the fluorescent dye ATT0594

292 ATTO594-Coenzyme A was custom-synthesised by Shinsei Kagaku, by conjugating 293 ATTO594-maleimide (ATTO-TECH) to Coenzyme A-SH (New England Biolabs), followed by 294 HPLC purification. The chemical structure of ATTO594-Coenzyme A was confirmed by liquid 295 chromatography-mass spectrometry (LC-MS; LCMS-2010A EV, Shimadzu). The ACP-tagged $296 \quad \beta 2$ AR expressed in the PM of the L cells was labelled with ATTO594, by incubating the cells 297 with $2 \mu \mathrm{M}$ ATTO594-Coenzyme A and $1 \mu \mathrm{M}$ phosphopantetheine transferase (New England 298 Biolabs) in Ham's F12 medium without FBS at $\sim 25^{\circ} \mathrm{C}$ for 20 min. These conditions have 299 been known to achieve 95\% labelling of ACP25. The cells were washed with P-HBSS three 300 times, and then subjected to microscope observations. The cells exhibiting the presence of 301 typically 1 ATTO594-ACP-B2AR spot/ $\mu \mathrm{m}^{2}$ in the PM were used for single-molecule imaging 302 experiments.

\section{Single fluorescent-molecule video imaging of live cells}

305 The fluorescently-labelled ACP- $\beta 2$ AR expressed in the bottom PM was observed at the level 306 of single molecules at $37^{\circ} \mathrm{C}$, using home-built objective-lens-type total internal reflection 307 fluorescence microscopes based on Olympus IX-70, Olympus IX-81, or Nikon TE-2000 308 inverted microscopes $4,28-30$, with a $100 x$ objective lens, as optimised for the present research. 309 Fluorescence images were projected onto a two-stage microchannel plate intensifier 310 (C8600-03, Hamamatsu Photonics), coupled to an electron bombardment CCD camera 311 (C7190-23; Hamamatsu Photonics) or an sCMOS camera (ORCA-Flash 4.0; Hamamatsu 312 Photonics) operated at $30 \mathrm{~Hz}$. For observations at a frame rate of $250 \mathrm{~Hz}$, a CMOS camera 313 (FASTCAM 1024PCI-II, Photron) was employed ${ }^{31}$. Fluorescent spots were identified by using 
314 an in-house computer program, as described previously ${ }^{4}$ The positions ( $x$ and $y$

315 coordinates) of all of the observed single fluorescent-molecules were determined by a 316 computer program that employed the method developed previously 32,33 . For the

317 simultaneous dual-color single-molecule imaging, the images were spectrally split into two 318 emission arms, each containing the same image intensifier and camera.

319 To maintain the cells at $37^{\circ} \mathrm{C}$ during the microscope observations, the entire 320 microscope, except for the two detection arms and the excitation arm, was placed in a 321 home-built microscope environment chamber made with thermo-insulating transparent 322 vinyl sheets, and three heating circulators (SKH0-112-OT, Kokensya) were appropriately 323 placed in the chamber to supply warmed air with slow circulation $30,34,35$. For further 324 stabilisation, a plate heater (INUG2, Tokai Hit) was mounted on the microscope stage. The 325 temperature of the surrounding air just above the stage was continually monitored during 326 the observations with a digital thermometer (DT-312, Intermedical).

Detection of colocalisation-codiffusion, and evaluations of $\boldsymbol{\beta} 2 \mathrm{AR}$ dimer lifetimes and two-dimensional dissociation constants of $\beta 2 A R$ dimers

330 The number densities of $\beta 2$ AR molecules existing as (true) dimers and the number density 331 of the expressed $\beta 2 A R$ molecules were determined by single-molecule imaging of ATTO594-ACP- $\beta 2 A R^{4}$. Colocalisations of two fluorescent spots (molecules) of the same color 333 (the same ATTO594 probe) or those of two different colors (YFP for BiFC and ATTO594) 334 were detected when the two fluorescent molecules became localised within $220 \mathrm{~nm}$ of each other ${ }^{4,30}$. The number densities of incidental colocalisations at given molecular densities were subtracted ${ }^{4}$. The two-dimensional dissociation constant (2D- $\left.K_{D}\right)$ of $\beta 2 A R$ in the PM was determined by using the method previously developed by us ${ }^{4}$.

To directly detect the actual binding of two $\beta 2 A R$ molecules, forming homo-dimers, we 339 employed the bimolecular fluorescence complementation (BiFC) method ${ }^{4,36}$. The details of 340 the BiFC method are described in the next subsection. 
To determine the lifetime of $\beta 2 A R$ dimers using single color observations (with ATTO594-ACP- $\beta 2 A R)$, the duration of each colocalisation-codiffusion event was measured 411,29 , and after the durations of many events were determined, histograms showing the distribution of colocalisation durations were obtained. All of the histograms obtained from the image sequences obtained at a time resolution of $33 \mathrm{~ms}$ could be fitted by single exponential functions, which provided decay time constants (lifetime; $\tau_{\text {observed }}$ ). $\tau_{\text {observed }}$ was then corrected for the photobleaching lifetime of the fluorescent probe ATTO594 ( $\tau_{\text {bleach }}=7.21 \pm 0.81 \mathrm{~s}$ for $30 \mathrm{~Hz}[n=300]$ and $0.812 \pm 0.041 \mathrm{~s}$ for $250 \mathrm{~Hz}[n=539]$ ), to

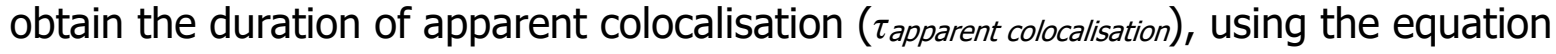
$\left[\tau_{\text {observed }^{-1}}-2 \tau_{\text {bleach }^{-1}}\right]^{-1} 4,11,29$. The effective dimer lifetime or the effective binding lifetime of 351 proteins, $\tau$, was evaluated by subtracting the duration of incidental colocalisation (without

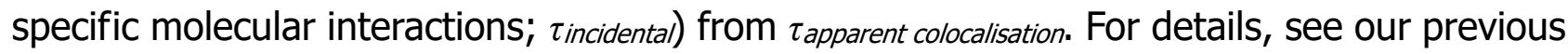

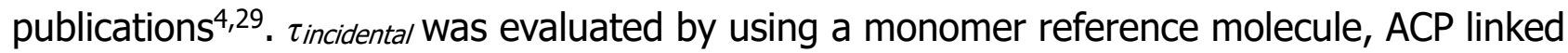
to the transmembrane domain of the low-density lipoprotein receptor, called ACP-TM ${ }^{29}$, and was found to be $19 \pm 0.46 \mathrm{~ms}$ (exponential lifetime; $n=241$ examined dimers; 39 examined movies; No. of Freedoms $=97$ ).

\section{Observations of the recruitment of $\mathbf{G} \alpha$ s and $\mathbf{G} \beta \gamma$ to $\boldsymbol{\beta A R}$ monomers and dimers}

359 The recruitment of $\mathrm{G} \alpha \mathrm{s}$ and $\mathrm{G} \beta \gamma$ to $\beta A R$ monomers and dimers was detected by observing

360 the colocalisation-codiffusion of mGFP-conjugated G $\alpha$ s and G $\beta \gamma$ with ATTO594-labelled $361 \quad \beta 2 A R$ monomers and dimers, at the level of single molecules. The colocalisation events lasting for longer than $66 \mathrm{~ms}$ (2 video frames) were analysed. The frequency of incidental colocalisation was estimated by superimposing an image sequence with one color on another image sequence with a different color that was artificially shifted by $\sim 500 \mathrm{~nm}$

365 (horizontally), and was subtracted. The recruitment frequencies of Gas and G $\beta \gamma$ to $\beta A R$ monomers and dimers were normalised by the number densities of G $\alpha$ s (or G $\beta \gamma$ ) and $\beta 2 A R$ protomers in the PM (per sec per $\mu \mathrm{m}^{4}$ ). 
The BiFC method for examining the formation of $\beta 2 A R$ dimers (the interaction of two $\beta 2 A R$ molecules at the molecular level)

371 The YFP-based $\mathrm{BiFC}(\mathrm{N})$ and $\mathrm{BiFC}(\mathrm{C})$ moieties employed previously ${ }^{4,37}$ were respectively

372 fused to the $C$ terminus of ACP- $\beta 2 A R$ (Extended Data Fig. 1) in the pEGFP-N1 vector

373 (Invitrogen; the cDNA region encoding EGFP was removed). These proteins were

374 co-expressed in L cells, by transfection with LipofectAMINE LTX (Life Technologies)

375 according to the manufacturer's recommendations, and then ATTO594 was conjugated to 376 the ACP moieties of ACP- $\beta 2 A R-B i F C(N)$ and ACP- $\beta 2 A R-B i F C(C)$. As the monomer reference 377 molecule for the BiFC data, ACP-TM was employed and the YFP-based BiFC(N) and BiFC(C) 378 moieties were fused to the $\mathrm{C}$ terminus of ACP-TM, as previously described ${ }^{4}$. Using dual-color 379 single-molecule imaging (ATTO594 and mGFP), the number densities of the expressed 380 molecules (the sum of the number densities of ACP-conjugated molecules) and those of the 381 fluorescence spots of reconstituted BiFC molecules (spots $/ \mu \mathrm{m}^{2}$ ) were determined.

\section{Evaluation of the changes of the cytosolic cAMP levels}

384 The amounts of cAMP in the cytosol were measured by using the time-resolved fluorescence resonance energy transfer (TR-FRET) kit from Perkin Elmer (EnVision Xcite), according to the manufacturer's recommendations. Since the $L$ cells used for single-molecule experiments expressed very low levels of $\beta 2 A R$, and thus the basal level of cAMP in the resting state was below the detection limit, we first established a stable L-cell line expressing $\beta 2 A R$ at high levels ${ }^{38}$, and used these cells to evaluate the changes of the cAMP levels in the cytosol before and after the additions of the agonist and inverse agonists. The drug treatments were performed as described at the end of the subsection, "Cell culture, cDNA construction and expression in L cells, and treatment of cells with agonists and inverse agonists". 
system based on the FK506-binding protein (FKBP)-ligand interface ${ }^{39}$. First, the cDNA encoding Halo7- $\beta 2$ AR-FKBP_F36V ( $\beta 2 A R-F K B P)$ was cloned into the expression plasmid vector pEGFP-N1 (Clontech) after the removal of the cDNA region encoding EGFP, and then the cDNA encoding FKBP_F36V was replaced by the cDNA encoding FKBP (obtained from the YFP-FKBP cDNA, the Addgene plasmid 20175 donated by T. Meyer ${ }^{40}$ ). Halo7- $\beta 2 A R-F K B P$ expressed in L cells was fluorescently labelled by incubating the cells for $24-48 \mathrm{~h}$ with $5 \mathrm{nM}$ Halo-tag conjugated with SaraFluor 650T (Goryo-Kayaku;

404 SaraFluor650T-Halo7-32AR-FKBP). The crosslinker for FKBP, AP20187 (Takara), was added 405 at a final concentration of $5 \mathrm{nM}$ during the image acquisition, and dimer formation was 406 detected at the level of single molecules.

For $\mathrm{Ca}^{2+}$ mobilisation assays, a freshly-prepared $1 \mathrm{mg} / \mathrm{ml}$ DMSO solution of Fluo4-AM (Dojindo) was added to HBSS buffered with $10 \mathrm{mM}$ (4-(2-hydroxyethyl)-1-piperazineethanesulfonic acid) at $\mathrm{pH} 7.4$ (H-HBSS), containing 1.25 mM Probenecid (Dojindo) and $0.04 \%$ (w/v) Pluronic F127 (Dojindo) (loading buffer), to

411 obtain a final Fluo4-AM concentration of $5 \mu \mathrm{g} / \mathrm{ml}(4.6 \mu \mathrm{M})$, basically following the

412 manufacturer's recommendations. The $L$ cells prepared as above for detecting dimers of

413 SaraFluor650T-Halo7- $\beta 2 A R-F K B P$ at the single-molecule level were incubated with the 414 loading buffer at $37^{\circ} \mathrm{C}$ for $1 \mathrm{~h}$ and washed with $\mathrm{H}$-HBSS three times, and then $500 \mu \mathrm{l}$ H-HBSS was added for epi-fluorescence microscopic observations. Fluo4 fluorescence was recorded at $2 \mathrm{~Hz}$. The stable dimers of SaraFluor650T-Halo7- $\beta 2 A R-F K B P$ were induced by

417 the addition of 5 nM AP20187, as described above. To determine the saturation levels of the 418 fluorescence signal intensity of Fluo4 at higher concentrations of $\mathrm{Ca}^{2+}, 1 \mu \mathrm{M}$ (final concentration) ionomycin (Wako) was added to the cells to equalise the intracellular $\mathrm{Ca}^{2+}$

420 concentrations with that outside the cells $(1.3 \mathrm{mM})$.

\section{Software and statistical analysis}

423 Superimpositions of image sequences obtained from dual-color single fluorescent molecule 424 observations and single fluorescent spot tracking were performed using C++-based 
425 computer programs produced in-house, as described previously ${ }^{30,32}$. Statistical analysis was 426 performed by the two-sided Welch's t test (except for the data shown in Extended Data 427 Fig. 5c, where the one-sided t test was used) or the Brunner-Munzel statistical test, by 428 using the $\mathrm{R}$ Studio Software ( $\mathrm{R}$ Foundation for Statistical Computing, 429 https://www.R-project.org/). P values less than 0.05 were considered to be statistically 430 significant. Linear and non-linear curve fittings were performed by using OriginPro 9.1 for 431 Windows, with appropriate scripts.

432

\section{Data and code availability}

434 All data generated or analysed for this study are available within the paper and its

435 associated Extended Data Figures and Tables. All other data presented and software codes 436 are available upon request from the corresponding author. A Reporting Summary for this 437 paper is available. 


\section{References}

4421 Nakashima, A. et al. Agonist-independent GPCR activity regulates anterior-posterior targeting of olfactory sensory neurons. Cel/ 154, 1314-1325 (2013).

4442 Seifert, R. \& Wenzel-Seifert, K. Constitutive activity of G-protein-coupled receptors: cause of disease and common property of wild-type receptors. Naunyn-Schmiedeberg's Arch. Pharmacol. 366, 381-416 (2002).

4473 Wacker, D., Stevens, R.C. \& Roth, B.L. How ligands illuminate GPCR molecular pharmacology. Cel/ 170, 414-427 (2017).

Kasai, R.S. et al. Full characterization of GPCR monomer-dimer dynamic equilibrium by single molecule imaging. J. Cell Biol. 192, 463-480 (2011).

4515 Calebiro, D. et al. Single-molecule analysis of fluorescently labeled G-protein-coupled receptors reveals complexes with distinct dynamics and organization. Proc. Natl. Acad. Sci. USA 110, 743-748 (2013). Hern, J.A. et al. Formation and dissociation of M1 muscarinic receptor dimers seen by total internal reflection fluorescence imaging of single molecules. Proc. Natl. Acad. Sci. USA 107, 2693-2698 (2010).

Tabor, A. et al. Visualization and ligand-induced modulation of dopamine receptor dimerization at the single molecule level. Sci. Rep. 6, 33233 (2016). James, J.R., Oliveira, M.I., Carmo, A.M., Iaboni, A. \& Davis, S.J. A rigorous experimental framework for detecting protein oligomerization using bioluminescence

10 Tennenberg, S.D., Zemlan, F.P. \& Solomkin, J.S. Characterization of $\mathrm{N}$-formyl-methionyl-leucyl-phenylalanine receptors on human neutrophils. Effects of isolation and temperature on receptor expression and functional activity. J. Immunol. 
141, 3937-3944 (1988).

47011 Suzuki, K.G. et al. Transient GPI-anchored protein homodimers are units for raft organization and function. Nat. Chem. Biol. 8, 774-783 (2012).

12 Meyer, B.H. et al. FRET imaging reveals that functional neurokinin-1 receptors are monomeric and reside in membrane microdomains of live cells. Proc. Natl. Acad. Sci. , 2138-2143 (2006)

47513 Katritch, V., Cherezov, V. \& Stevens, R.C. Structure-function of the G protein-coupled receptor superfamily. Annu. Rev. Pharmacool. Toxicol. 53, 531-556 (2013).

47714 Ballesteros, J.A. et al. Activation of the beta 2-adrenergic receptor involves disruption of an ionic lock between the cytoplasmic ends of transmembrane segments 3 and 6 . J. Biol. Chem. 276, 29171-29177 (2001).

O'Dowd, B.F. et al. Site-directed mutagenesis of the cytoplasmic domains of the human beta 2-adrenergic receptor. Localization of regions involved in $\mathrm{G}$ protein-receptor coupling. J. Biol. Chem. 263, 15985-15992 (1988).

Kahsai, A.W. et al. Multiple ligand-specific conformations of the beta2-adrenergic receptor. Nat. Chem. Biol. 7, 692-700 (2011).

19 Schmidt, M. et al. A new phospholipase-C-calcium signalling pathway mediated by cyclic AMP and a Rap GTPase. Nat. Cell Biol. 3, 1020-1024 (2001). Kalay, Z., Fujiwara, T.K. \& Kusumi, A. Confining domains lead to reaction bursts: reaction kinetics in the plasma membrane. PloS one 7, e32948 (2012).

DeVree, B.T. et al. Allosteric coupling from G protein to the agonist-binding pocket in GPCRs. Nature 535, 182-186 (2016).

Sungkaworn, T. et al. Single-molecule imaging reveals receptor-G protein interactions at cell surface hot spots. Nature 550, 543-547 (2017).

21 Kusumi, A. et al. Dynamic organizing principles of the plasma membrane that regulate signal transduction: commemorating the fortieth anniversary of Singer and Nicolson's fluid-mosaic model. Annu. Rev. Cell Dev. Biol. 28, 215-250 (2012). Kusumi, A. et al. Paradigm shift of the plasma membrane concept from the 
two-dimensional continuum fluid to the partitioned fluid: high-speed single-molecule tracking of membrane molecules. Annu. Rev. Biophys. Biomol. Struct. 34, 351-378 (2005).

50023 Davenport, A.P. et al. International Union of Basic and Clinical Pharmacology. LXXXVIII. G protein-coupled receptor list: recommendations for new pairings with cognate ligands. Pharmacol. Rev. 65, 967-986 (2013).

50324 Dixon, R.A. et al. Ligand binding to the beta-adrenergic receptor involves its rhodopsin-like core. Nature 326, 73-77 (1987).

50525 George, N., Pick, H., Vogel, H., Johnsson, N. \& Johnsson, K. Specific labeling of cell surface proteins with chemically diverse compounds. J. Am. Chem. Soc. 126, 8896-8897 (2004).

50826 Yu, J.Z. \& Rasenick, M.M. Real-time visualization of a fluorescent G(alpha)(s): dissociation of the activated G protein from plasma membrane. Mol. Pharmacol. 61, 352-359 (2002).

51127 Janetopoulos, C., Jin, T. \& Devreotes, P. Receptor-mediated activation of heterotrimeric G-proteins in living cells. Science 291, 2408-2411 (2001).

51328 Iino, R., Koyama, I. \& Kusumi, A. Single molecule imaging of green fluorescent proteins in living cells: E-cadherin forms oligomers on the free cell surface. Biophys. J. 80, 2667-2677 (2001).

51629 Kasai, R.S., Ito, S.V., Awane, R.M., Fujiwara, T.K. \& Kusumi, A. The class-A GPCR 517 dopamine D2 receptor forms transient dimers stabilized by agonists: detection by single-molecule tracking. Cell Biochem. Biophys. 76, 29-37 (2018). Koyama-Honda, I. et al. Fluorescence imaging for monitoring the colocalization of two single molecules in living cells. Biophys. J. 88, 2126-2136 (2005).

$52131 \quad$ Hiramoto-Yamaki, N. et al. Ultrafast diffusion of a fluorescent cholesterol analog in 522 compartmentalized plasma membranes. Traffic 15, 583-612 (2014).

52332 Fujiwara, T., Ritchie, K., Murakoshi, H., Jacobson, K. \& Kusumi, A. Phospholipids undergo hop diffusion in compartmentalized cell membrane. J. Cell Biol. 157, 
1071-1081 (2002).

52633 Gelles, J., Schnapp, B.J. \& Sheetz, M.P. Tracking kinesin-driven movements with nanometre-scale precision. Nature 331, 450-453 (1988).

52834 Murakoshi, H. et al. Single-molecule imaging analysis of Ras activation in living cells. Proc. Natl. Acad. Sci. USA 101, 7317-7322 (2004).

53035 Tsunoyama, T.A. et al. Super-long single-molecule tracking reveals dynamic-anchorage-induced integrin function. Nat. Chem. Biol. 14, 497-506 (2018).

$36 \mathrm{Hu}$, C.D., Chinenov, Y. \& Kerppola, T.K. Visualization of interactions among bZIP and Rel family proteins in living cells using bimolecular fluorescence complementation. Mol. Cel/ 9, 789-798 (2002).

53537 Briddon, S.J. \& Hill, S.J. Pharmacology under the microscope: the use of fluorescence correlation spectroscopy to determine the properties of ligand-receptor complexes. Trends Pharmacol. Sci. 28, 637-645 (2007).

38 Yusa, K., Zhou, L., Li, M.A., Bradley, A. \& Craig, N.L. A hyperactive piggyBac transposase for mammalian applications. Proc. Natl. Acad. Sci. USA 108, 1531-1536 (2011).

54139 Clackson, T. et al. Redesigning an FKBP-ligand interface to generate chemical dimerizers with novel specificity. Proc. Natl. Acad. Sci. USA 95, 10437-10442 (1998).

54340 Inoue, T., Heo, W.D., Grimley, J.S., Wandless, T.J. \& Meyer, T. An inducible translocation strategy to rapidly activate and inhibit small GTPase signaling pathways. Nat. Methods 2, 415-418 (2005). 


\section{Fig. 1}
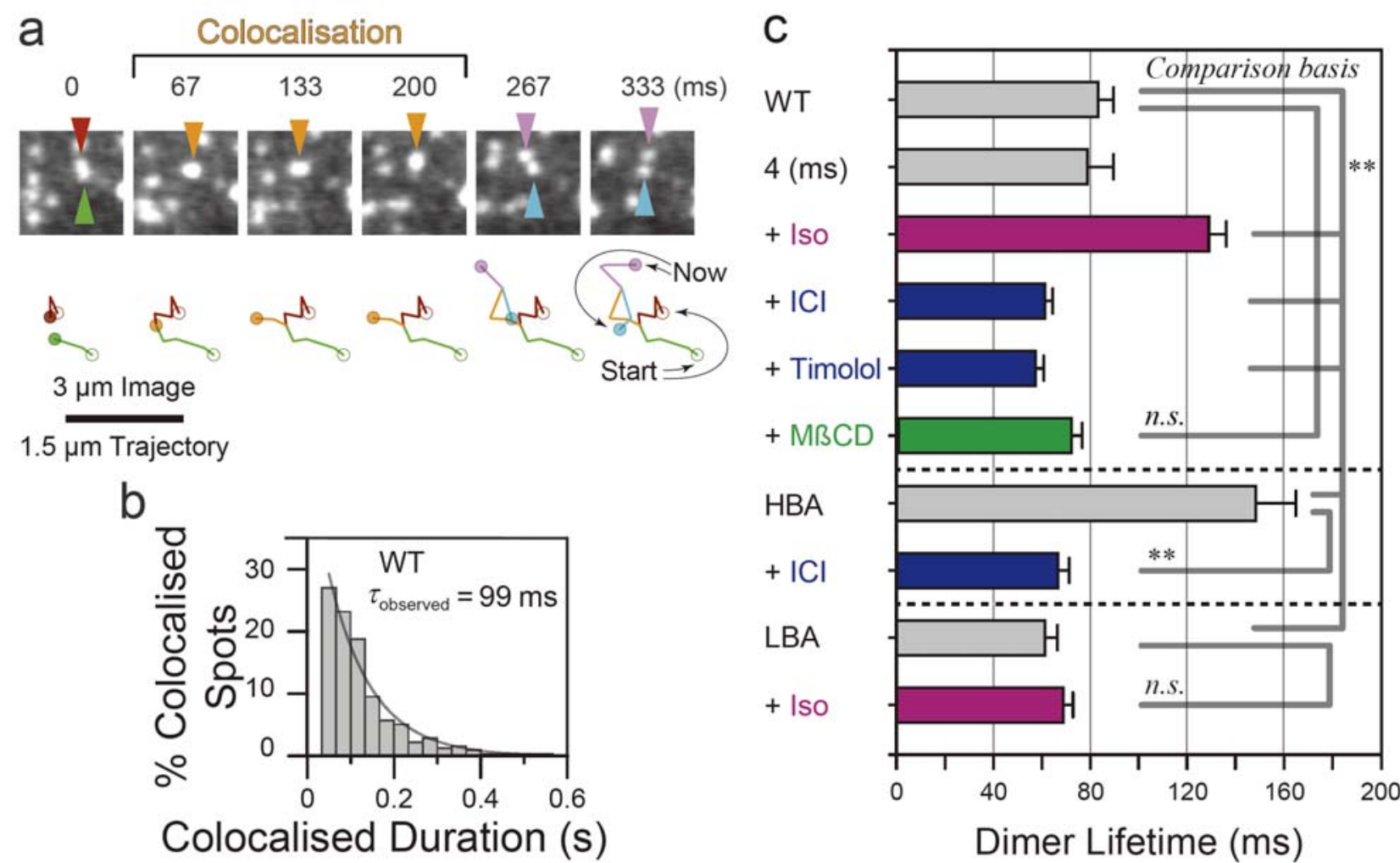

Dimer Lifetime (ms)

Fig. 1. $\beta 2 A R$ dynamically interconverts between monomers and transient homodimers.

Mean, SEM, the number of experiments, and other statistical parameters are summarised in

\section{Extended Data Table 1.}

(a) A typical snapshot sequence showing transient homo-dimerisation of $\beta 2 A R$ molecules. Two molecules became colocalised at frame $2(67 \mathrm{~ms})$, diffused together for $133 \mathrm{~ms}$ (colocalisation), and then separated.

(b) Histogram showing the distribution of the durations of the WT- $\beta 2$ AR colocalisation events (homo-dimers). Note that the $\tau_{\text {observed }}$ is the value before corrections for photobleaching and incidental colocalisations.

(c) Summary of the dimer lifetimes (after corrections) of WT and mutant $\beta 2 A R$ obtained before and after the additions of various reagents. ${ }^{* *}$ and $n$.s.: statistically significant and non-significant with $P$ values smaller or greater than 0.05 , respectively. This convention is used throughout this report. 


\section{Fig. 2}

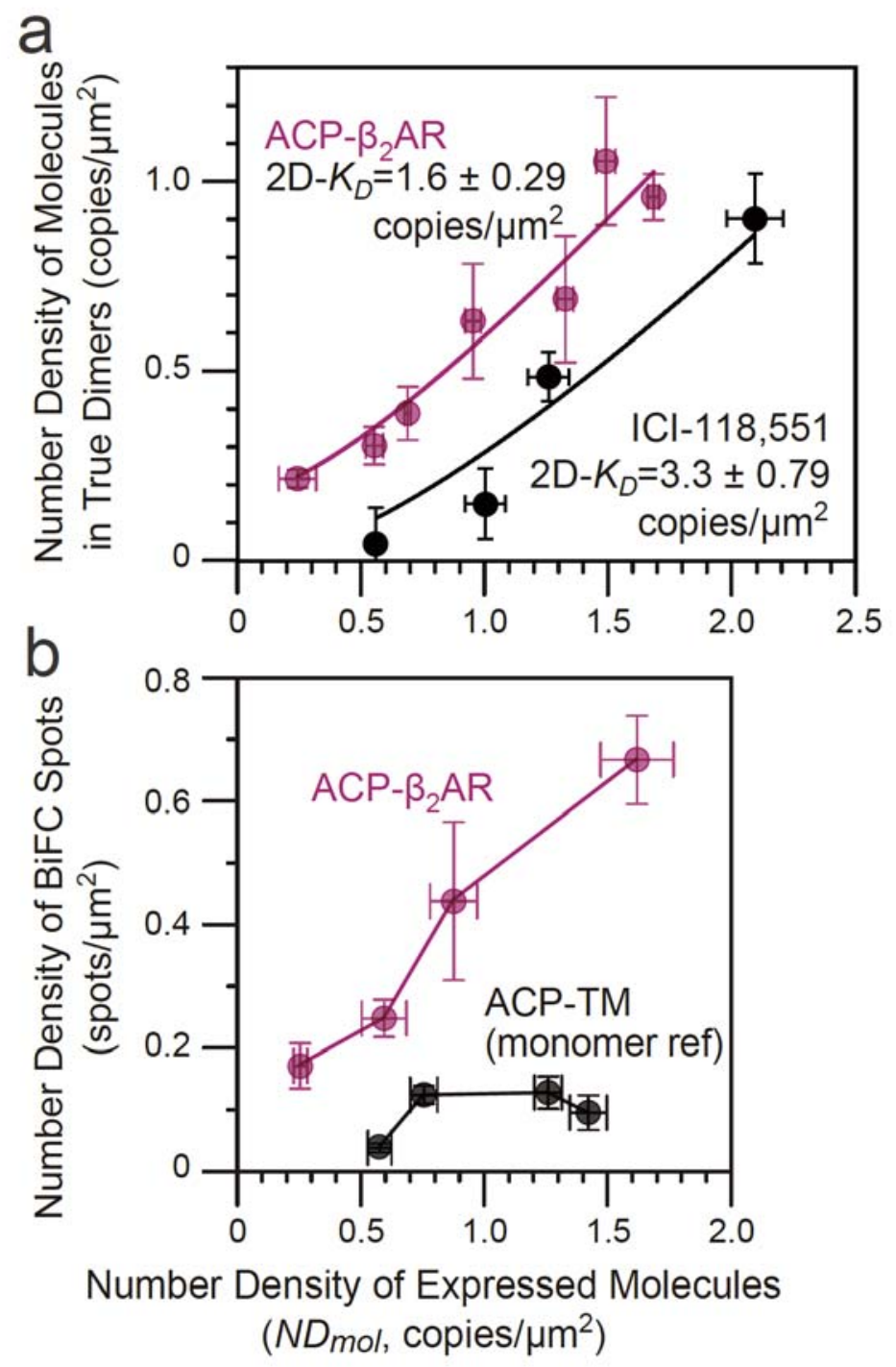

Fig. 2. Determining the 2D-KD of $\beta 2 A R$ dimers with/without an inverse agonist and the BiFC detection of $\boldsymbol{\beta 2 A R}$ dimers. Mean, SEM, the number of experiments, and other statistical parameters are summarised in Extended Data Table 2.

The number density of $\beta 2 A R$ molecules in true dimers (after subtraction of incidental overlap of fluorescent spots; \pm inverse agonist ICI-118,551; curves represent the fitting results using Eq. 5 in Ref. 4) (a) and the number densities of the BiFC spots for ACP- $\beta 2 A R-Y F P(N / C)$ and ACP-TM-YFP(N/C) (monomer reference molecule) (b), plotted as a function of the number density of expressed molecules (NDmol). In $\mathbf{b}$, the lines are provided to facilitate visualisation. 


\section{Fig. 3}

a

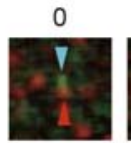

Start

$3 \mu \mathrm{m}$ Image

$0.6 \mu \mathrm{m}$ Trajectory
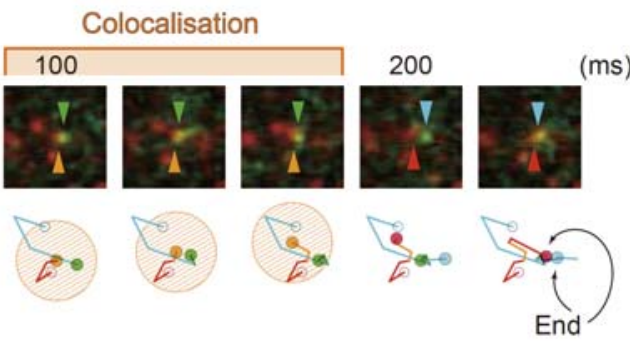

b $G a$

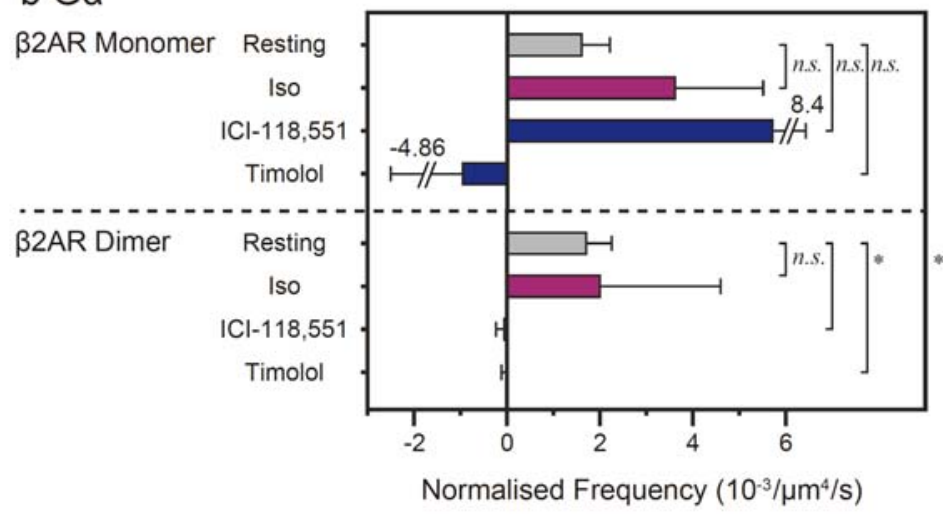

c $G \beta$

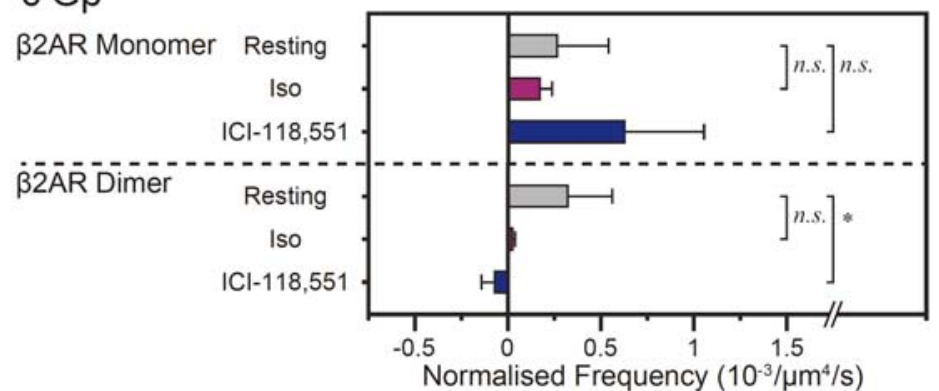

Fig. 3. Non-stimulated $\beta 2 A R$ homo-dimers recruit trimeric G-proteins: a process

suppressed by inverse agonists. Mean, SEM, the number of experiments, and other statistical parameters are summarised in Extended Data Table 3.

(a) A typical snapshot image sequence showing that a trimeric G-protein Gas subunit transiently binds to a $\beta 2 A R$ monomer.

(b, c) The inverse agonists, ICI-118,551 and timolol, inhibitors of $\beta 2 A R$ basal constitutive activities, blocked the recruitment of trimeric G-proteins to $\beta 2 A R$ homo-dimers, but not that to monomers. G $\alpha$ s (b) and $\mathrm{G} \beta \gamma$ (c) colocalisation frequencies with $\beta 2 \mathrm{AR}$ monomers and homo-dimers, normalised by the number densities of $\mathrm{G} \alpha \mathrm{s}$ (or $\mathrm{G} \beta \gamma$ ) and $\beta 2 \mathrm{AR}$ protomers (per sec per $\mu \mathrm{m}^{4}$ ). 